\title{
Analysis of MIMO Capacity Dependence on Multi- Antenna Channel Delay Spread in Reverberation Chamber
}

\author{
N. Arsalane \\ Institut de Génie \\ Appliqué Casablanca \\ 41, Avenue 2 Mars \\ Casablanca, Maroc
}

\author{
M. A. Garcia- \\ Fernandez \\ Institut XLIM 127, \\ avenue Albert \\ Thomas \\ M. Mouhamadou \\ Institut XLIM 127, \\ avenue Albert \\ Thomas \\ Limoges, France
}

Limoges, France

\author{
C. Decroze \\ Institut XLIM 127, \\ avenue Albert \\ Thomas \\ Limoges, France
}

\author{
D. Carsenat \\ Institut XLIM 127, \\ avenue Albert \\ Thomas \\ Limoges, France
}

\begin{abstract}
Reverberation chambers are cost-effective tools for evaluating Multiple-Input Multiple-Output (MIMO) Over-The-Air (OTA) communication devices performance in both indoor and outdoor urban channel models. Their performance is typically assessed by using several figures-of-merit (FOM), such as MIMO Capacity, which evaluates the amount of information that can be reliably transmitted over a communications channel. Nevertheless, scarce publications about the effect of the environment characteristics, such as delay spread, on this figure-of-merit can be found in the literature. In this contribution, we analyze the dependence of MIMO Capacity on channel delay spread, by means of a multi-antenna OTA communication system using a QPSK modulation. For that purpose, channel RMS delay spread has been varied inside the reverberation chamber loading it with different quantities of absorbing material.
\end{abstract}

\section{Keywords}

Channel capacity; MIMO, Phase shift keying, Rayleigh channels, Reverberation chamber

\section{INTRODUCTION}

In the last few years, multiple-input multiple-output (MIMO) over-the-air (OTA) communication systems have drawn considerable attention due to their performance-enhancement capability in multipath environments [1]. Reverberation chambers are considered cost-effective tools for evaluating these MIMO OTA systems, which constitute a part of daily life nowadays, since they offer significant increases in data throughput and wireless link range without additional bandwidth or transmit power, by means of gathering several antennas [2]. In order to assess the data throughput associated to the communication channel, MIMO capacity is the figureof-merit (FOM) typically used to present its effectiveness. Assuming that the receiver has perfect channel state information and that transmitted power is equally distributed among transmitting antenna elements, the ergodic MIMO capacity can be expressed as $[1,3]$

$$
C_{N_{r} x N_{r}}=E\left\{\log _{2}\left[\operatorname{det}\left(I_{N_{r}}+\frac{\gamma}{N_{t}} H_{N_{r} x N_{t}} H_{N_{r} x N_{t}}^{H}\right)\right]\right\}
$$

where $\gamma$ is signal-to-noise ratio (SNR), the superscript $\mathrm{H}$ is Hermitian operator, HNrxNt is the channel matrix, $\mathrm{Nt}$ and $\mathrm{Nr}$ are the number of transmitting and receiving antennas, respectively, and $\mathrm{E}$ is statistical expectation taken over samples of the channel matrix describing the fading. HNrxNt is normalized so that its Frobenius norm satisfies $\left\|H_{N_{r} x N_{t}}\right\|_{F}^{2}=N_{r} \times N_{t}$

[4]. Despite the importance of MIMO capacity as figure-of-merit in multi-antenna OTA communication systems, scarce publications can be found in the literature related to how it is affected by the channel characteristics. Thus, MIMO capacity dependence on the channel characteristics must be analyzed for better understanding of MIMO OTA passive measurements for device characterization. MIMO OTA channels are usually characterized by their coherence distance, time and bandwidth, or equivalently by their reciprocal counterparts: angular, Doppler and delay spread, respectively $[1,5]$. In order to simplify the analysis, a frequency-flat Rayleigh fading channel has been selected for this study. Hence, in this contribution, we present a study of the MIMO capacity dependence on channel characteristics by means of both simulations and measurements. Also, a reverberation chamber has been used in order to emulate the Rayleigh fading channel for carrying out the measurements needed to validate the simulation results. MIMO capacity has been found to be in direct variation to the reverberation chamber channel powerdelay profile RMS delay spread when different amounts of absorbing material are placed inside the chamber inner cavity. The reason for this is discussed along the following sections.

\section{MEASUREMENT PLATFORM}

The experimental platform for MIMO capacity measurement consists of a PXI chassis and is described in Figure 1. The transmitter integrates a controller and is made of a base band signal generator with modulation mapping, which is sent to the RF front-end. The signal generator provides IQ modulation and arbitrary waveform generation, up to $6 \mathrm{GHz}$. The filtered IQ symbols with root raised cosine filter are transmitted through the propagation channel via the transmitter antenna. The receiver integrates two triggered $\mathrm{RF}$ digitizers to perform the conversion of the received RF signal to the digital IQ data. The IQ data are processed with a MATLAB program via the PXI controller. For the measurements presented here, a short bandwidth of $100 \mathrm{kHz}$ has been chosen over a carrier frequency of $5.5 \mathrm{GHz}$, collecting 32000 samples for each measurement. 


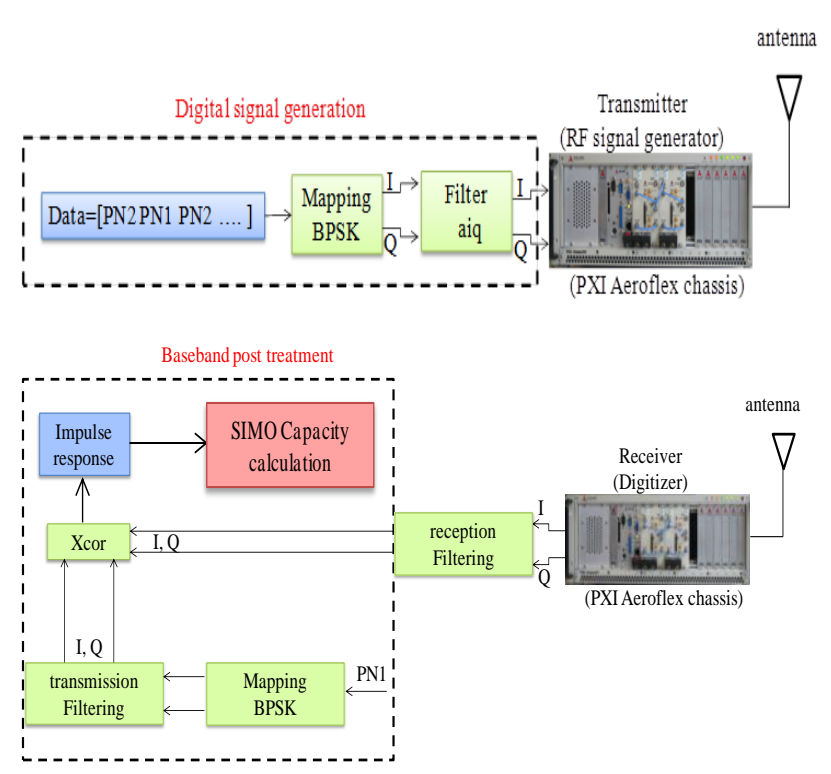

Figure1: Channel sounding measurement platform (SISO case)

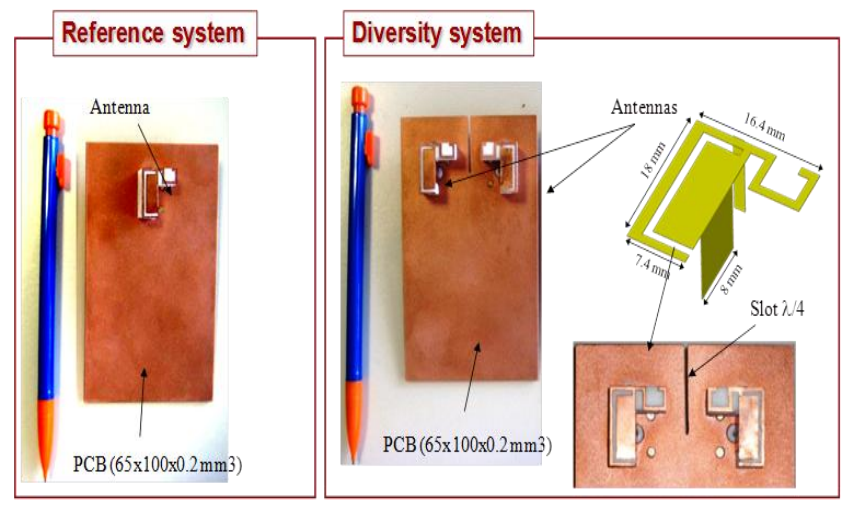

Figure2: Compact wideband triband antennas for mobile terminals based on planar inverted-F antenna (PIFA)

The reverberation chamber is excited by two horn antennas connected to the transmitter, which integrates the controller and Aeroflex modular RF signal generator with an integrated dual-channel arbitrary waveform generator, and with a modulation bandwidth up to $66 \mathrm{MHz}$. The antennas under test are compact wideband triband antennas for mobile terminals [12], based on planar inverted-F antenna (PIFA) elements, as depicted in Fig. 3.

In order to study the dependence of the MIMO capacity performance on the RMS delay spread of the multi-path channel in the RC, different amount of absorbing material have been introduced inside its inner cavity, thus modifying the power delay profile (PDP) of the channel, and achieving scenarios with different RMS delay spread values, as depicted in Fig.3.

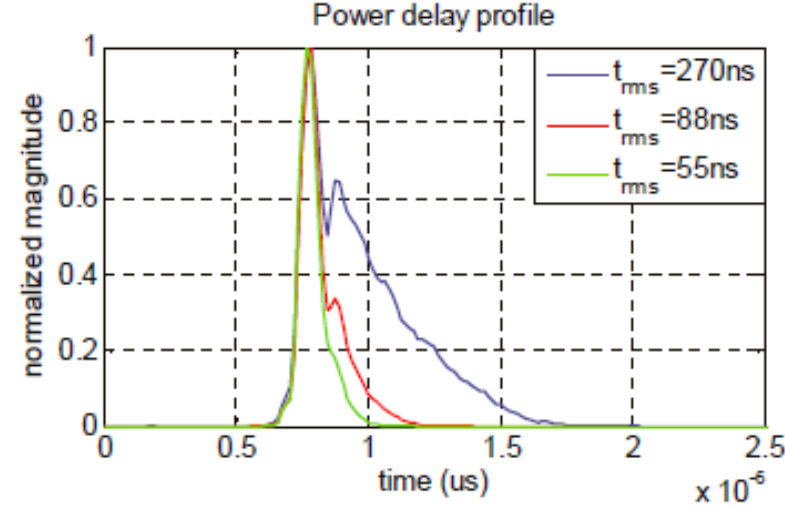

Figure 3: Measured PDPs for different RMS delay spread values

The results in Fig.3 highlight the dependence of the RMS delay spread measured value on the amount of absorbing material located inside the RC. The use of absorbing material decreases considerably the RMS delay spread value [10,11].

Since the introduction of absorbing materials also increases the power losses, a different transmitted power has been chosen for each scenario, in order to compensate these losses, and thus obtaining the same average power at the diversity antennas (Receiver) for each scenario, in order to make the MIMO capacity results directly comparable.

\section{MIMO CAPACITY TEST BED}

The experimental platform for MIMO capacity described in Figure (1) comprises two independent PXI chassis. The transmitter integrates a controller and is made of a base band signal generator with modulation mapping, which is sent to the RF front end. The signal generator provides IQ modulation and arbitrary wave form generation, up to $6 \mathrm{GHz}$. The filtered IQ symbols with root raised cosine filter are transmitted through the propagation via the transmitter antenna. The receiver integrates two triggered $\mathrm{RF}$ digitizers to perform the conversion of the received RF signal to the digital IQ data. This measurement technique uses the autocorrelation properties of PN sequences. The transmitted PN code will be correlated with the PN code generated at the reception. This correlation made before data acquisition is performed by a correlator (mixer and an integrator). The correlation step is deferred to a post digital processing Matlab. The generation of the PN sequence and shaping of data are performed in Matlab. The transmitted PN sequence is the result of concatenating two sequences PN1 and PN2 having the same length. The PN sequence generator consists of $r$ registers. The length of a sequence is given by $N_{c}=2^{r}-1$. In our case, we generated the two sequences PN1 and PN2 with 9 registers, making a length of 511 bits for each sequence. A shaping of this sequence is to perform a BPSK modulation followed by filtering root raised cosine to limit the signal band $\mathrm{PN}$ issued. Thus, the PN sequence is sampled at a frequency of $66 \mathrm{MHz}$. A created text file in Matlab contains the values of each sample of the PN sequence filtered and BPSK modulated. This file is converted to readable format aiq by RF generator of emission module.

This file contains the sequence to be transmitted in the baseband. Then, the generator sends the PN sequence on a carrier frequency of $5.5 \mathrm{GHz}$. At the reception, the digitizer translates the RF signals to intermediate frequency of $77 \mathrm{MHz}$ 
to be digitized. The baseband processing at the reception is to achieve a filter on the IQ quadrature signals so as to minimize the noise band. The filter used has the same impulse response as in emission. THE SIMO capacity, based on impulse response estimation algorithm is implemented.

In this contribution, we present an analysis of the dependence of MIMO capacity on the wireless channel characteristics. Different RMS delay spread profiles have been achieved by varying the amounts of absorbers placed within the reverberation chamber cavity.

When a reverberation chamber is sampled step-wisely, i.e. in static condition at each stirrer position, the Doppler spread of the channel is negligible.

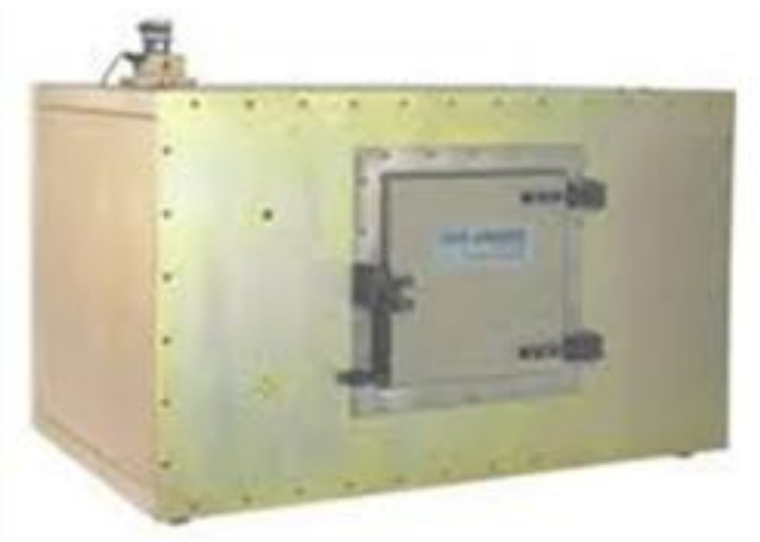

Figure4: Mode Stirred Reverberation Chamber

\section{MIMO CAPACITY SIMULATION AND MEASUREMENT IN REVERBERATION CHAMBER}

The previously presented system is used to evaluate SIMO capacity in a mode-stirred reverberation chamber (RC using the sounding channel measurement test-bed). A reverberation chamber is a closed metal cavity with dimensions larger than a few wavelengths over a wide frequency range. Electromagnetic fields inside (at a reasonable distance from the walls of the order of the wavelength) have a Statistical uniformity at all points of measurements in all directions and for all positions of the radiation source. This uniformity is obtained using the Mechanical (s) stirrer (s) rotating inside the chamber. The RC used for our measurement presents the following dimensions $(1 \mathrm{~m} \times 0.7 \mathrm{~m}$ $\mathrm{x} 0.6 \mathrm{~m}$ ) (Figure 4) and the measurements were performed at 3.5 GHz. This chamber is excited by a circular patch antenna. The patch antenna is connected to the transmitter PXI chassis and the tow antennas under test are connected in the receiver PXI chassis. The antennas under test are positioned in the test volume; our reverberation chamber has tow mechanical stirrers which are rotated to obtain a statistically isotropic channel.

The SIMO capacity is calculated from the estimation of the impulse response, by using the equation (1). The capacity curves for the different scenarios are plotted in (figure) versus $\mathrm{SNR}$. We can see that there is equivalence between simulations and measurements.

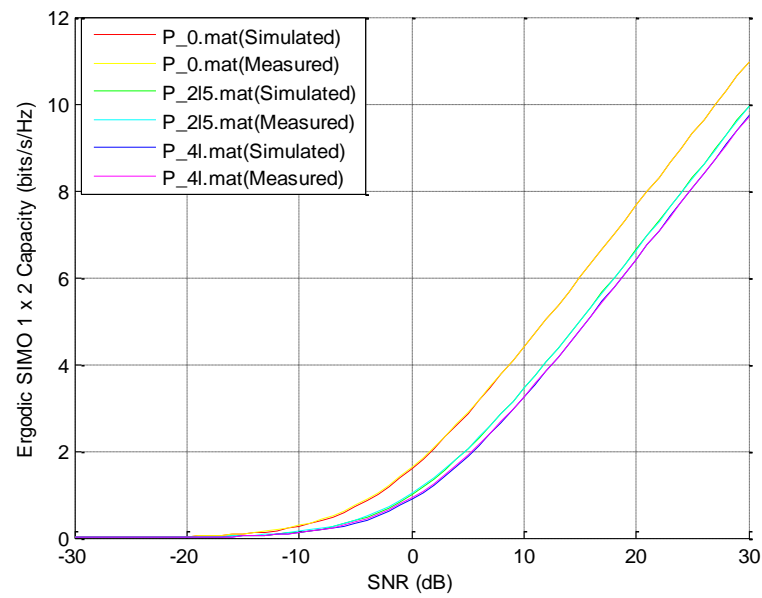

Figure5: Simulation and measurement for SIMO capacity versus SNR

The SIMO capacity concerning the first scenario when the chamber is empty (higher delay spread) presents a maximum capacity. And in the same way we can see that the system capacity decrease versus increasing the quantity of water inside the reverberation chamber (scenario $2 \& 3$ ).

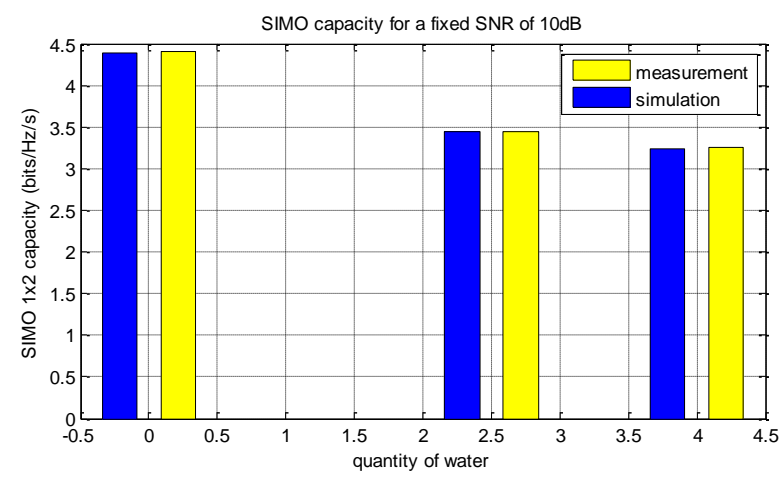

Figure6: Simulation and measurement for SIMO capacity versus quantity of water for fixed value of SNR

The results shown in Figure 4 show the simulation and measurement values of MIMO capacity for a fixed value of SNR that is equal to $10 \mathrm{~dB}$, measurements are made for three different cases, the first case the room is empty, the second case we introduce 2.5 liters of water, and the third and final case was introduced 41 of water. It is known that the introduction of these loss quantities: will affect the value of quality factor of the reverberation chamber and later that of the RMS delay spread value, and we can see this relationship from the following equation (2).

$$
\tau_{R M S}=\frac{Q}{2 \pi f}
$$

What we can see, that there is a relationship between the decreasing in the value of system capacity and the increasing of the amount of losses within the room (decrease delay spread).

\section{CONCLUSIONS}

MIMO capacity has been found to be in direct variation to the channel power-delay profile RMS delay spread when it is varied through the introduction of lossy objects, since MIMO 
capacity depends directly on the actual instantaneous transmitted power, as can be derived straightforwardly from (1), and this transmitted power is diminished when the inner cavity of the reverberation chamber is loaded with absorbing materials.

\section{REFERENCES}

[1] A. Paulraj, R. Nabar and D. Gore, Introduction to Spacetime Wireless Communication, Cambridge University Press, 2003.

[2] K. Fujimoto and J. R. James, Mobile Antenna Systems Handbook, chapter 2, Artech House, Norwood, MA, USA, 1994.

[3] C. J. Foschini, "Layered space-time architecture for wireless communication in a fading environment when using multi-element antennas," Bell Labs Tech. J., vol. 1, pp. 41-59, 1996.

[4] X. Chen, et al., "Comparison of Ergodic Capacities From Wideband MIMO Antenna Measurements in Reverberation Chamber and Anechoic Chamber", IEEE Antennas and Wireless Propagation Letters, vol.10, pp. 446-449, 2011

[5] X. Chen, "Spatial Correlation and Ergodic Capacity of MIMO Channel in Reverberation Chamber", International Journal on Antennas and Propagation, 2011.

[6] A. Milewski, Periodic sequences with optimal properties for channel estimation and fast start-up equalization, IBM J Res Develop, 37 (1983), 426-431.
[7] IEEE Standard for Local and metropolitan area networks-Part 16: Air Interface for Fixed Broadband Wireless Access Systems, IEEE Std 802.16-2004 (Revision of IEEE Std 802.16-2001).

[8] M. Mouhamadou et al., "Active Measurements of Antenna Diversity Performances Using a Specific TestBed, in Several Environments", International Journal of $\mathrm{RF}$ and Microwave Computer-Aided Engineering, pp. 264-271, Nov. 2009.

[9] O. Delangre, Ph. De Doncker, M. Lienard and P. Degauque, "Delay spread and coherence bandwidth in reverberation chamber", ELECTRONICS LETTERS $28^{\mathrm{TH}}$ February 2008 Vol. 44 No.

[10] E. Genender, C.L. Holloway, K.A. Rembey, J.M Ladbury, G. Koepke, H, Garbe, "Simulating the multipath channel with a reverberation chamber, application to bit error rate measurement," IEEE transactions on Electromagnetic compatibility, vol. 52, pp. 766-777, 2010.

[11] F. Y. Luan, Y. Zhang, X. W. Hu, S. D. Zhou, L. M. Xiao, X. B. Xu, "Measurement based delay spread analysis of indoor distributed antenna systems", Proceeding of the $5^{\text {th }}$ European Conference on antenna and Propagation (Eucap 2011), pp. 2556-2559, 2011.

[12] M. Koubeissi, M. Mouhamadou, C. Decroze, D. Carsenat, and T.Monédière, "Triband Compact Antenna for Multistandard Terminals and User's Hand Effect," International Journal of Antennas and Propagation, pp. 17, Dec. 72009. 